Pacific

Journal of

Mathematics

CLASSIFICATION OF STRONGLY FREE ACTIONS OF DISCRETE AMENABLE GROUPS ON

STRONGLY AMENABLE SUBFACTORS OF TYPE $I I I_{0}$

TOSHIHIKo MASUdA

Volume $191 \quad$ No. 2

December 1999 


\title{
CLASSIFICATION OF STRONGLY FREE ACTIONS OF DISCRETE AMENABLE GROUPS ON STRONGLY AMENABLE SUBFACTORS OF TYPE III
}

\author{
TOSHIHIKo MAsudA
}

\begin{abstract}
We classify the strongly free actions of discrete amenable groups on strongly amenable subfactors of type III $_{0}$. Winsløw's fundamental homomorphism is a complete invariant.
\end{abstract}

\section{Introduction.}

In the theory of operator algebras, classification of group actions on approximately finite dimensional (AFD) factors has been done since Connes's work $[2]$.

In subfactor theory, various results on classification of group actions have been obtained. The most powerful results have been obtained by Popa in [16], who classified the strongly outer actions of discrete amenable groups on strongly amenable subfactors of type $\mathrm{II}_{1}$ up to cocycle conjugacy. (Strong outerness for automorphisms are introduced by Choda-Kosaki in [1], and Popa in [16] independently. Popa use the terminology "proper outerness".)

In our previous work [13], we have classified the strongly free actions of discrete amenable groups on strongly amenable subfactors of type $\mathrm{III}_{\lambda}$, $0<\lambda<1$. Our method in [13] has been based on [18] and [19]. But in [18] and [19], Sutherland and Takesaki treated factors of type $\mathrm{III}_{\lambda}, 0 \leq \lambda<1$, including the case $\lambda=0$. So it is natural to ask if their method works for the classification of group actions on subfactors of type $\mathrm{III}_{0}$. In this paper, we classify strongly free actions of discrete amenable groups on strongly amenable subfactors of type $\mathrm{III}_{0}$. The complete invariant we use is Winsløw's fundamental homomorphism, [22, Definition 4.2], which is an analogue of the Connes-Takesaki module ([5]) in subfactor theory. It is well known that in the single factor case, centrally free actions of discrete amenable groups on injective factors are completely classified by their Connes-Takesaki modules, [2], [14], [18], [19], [10]. And in subfactor theory, strong freeness is an analogy of centrally freeness, so the results in [13] and this paper are "subfactor-version" of these results. (In the case of strongly amenable subfactors of type II and type $\mathrm{III}_{\lambda}, 0<\lambda<1$, strong freeness is equivalent to central freeness. See $[\mathbf{1 6}],[\mathbf{2 1}]$.) 
To classify actions on subfactors of type $\mathrm{III}_{0}$, we must consider actions of groupoids on subfactors of type $\mathrm{II}_{\infty}$. Groupoid actions on semifinite factors are studied in [9] first, and developed in [18] and [19]. A basic idea to classify groupoid actions is the followings. First we split a groupoid into an isotropy part and a principal part, second apply the classification results of group actions to the isotropy part, and the cohomology lemma to the principal part, and finally combine them. Our idea to classify groupoid actions on strongly amenable subfactor of type $\mathrm{II}_{\infty}$ owes much to this idea.

Note that in the type $\mathrm{III}_{\lambda}$ case, $0<\lambda<1$, we need only classification results on group actions and do not have to use the cohomology lemma because the form of the flow of weights of type $\mathrm{III}_{\lambda}$ factor is simple and the principal parts become trivial.

This paper is organized as follows.

In Section 2, we collect facts on group actions and strongly amenable subfactor of type $\mathrm{III}_{0}$ which we need in this paper.

In Section 3, we explain how to reduce the classification of group actions to that of groupoid actions. We also discuss relation between invariants of group actions and those of groupoid actions.

In Section 4, we classify groupoid actions on strongly amenable subfactor of $\mathrm{II}_{\infty}$. We split the groupoid into the isotropy part and the principal part. To classify the isotropy part, we use Popa's classification result, [16, Theorem 3.1] and to classify the principal part, we use the cohomology lemma, [17, Theorem 5.5]. Then we get outer conjugacy, but it may happen that the actions are not cocycle conjugate. To obtain cocycle conjugacy, we use model actions constructed by Sutherland and Takesaki in [18] and we complete the classification.

Acknowledgement. The author is grateful to Prof. M. Izumi and Prof. Y. Kawahigashi for helpful advice and constant encouragement. He also thanks Professor C. Winsløw for pointing out his misunderstanding in Theorem 2.7

\section{Preliminaries and notations.}

In this section, we recall several facts about group actions on subfactors and strongly amenable subfactors of type $\mathrm{III}_{0}$.

Definition 2.1 ([1, Definition 1], [16, Definition 1.5.1]). Let $N \subset M$ be a subfactor with finite index and $N \subset M \subset M_{1} \subset \cdots$ be the Jones tower. For $\alpha \in \operatorname{Aut}(M, N), \alpha$ is said to be strongly outer if there are no nonzero $a \in M_{k}$ such that $\alpha(x) a=a x$ holds for every $x \in M$. An action $\alpha$ of $G$ is said to be strongly outer if $\alpha_{g}$ is strongly outer for $g \in G \backslash\{e\}$.

Definition $2.2\left(\left[\mathbf{1 2}\right.\right.$, Section 5]). Set $\Phi(\alpha):=\left\{\left.\alpha\right|_{M^{\prime} \cap M_{k}}\right\}_{k}$. We call $\Phi(\alpha)$ the Loi invariant. 
In the case of subfactors in type II, the following classification results are obtained by Popa and Winsløw.

Theorem 2.3 ([16, Theorem 2.1, Theorem 3.1], [21, Theorem 4.3]). Let $N \subset M$ be a strongly amenable subfactor of type $\mathrm{II}_{1}$ or $\mathrm{II}_{\infty}$. Let $\alpha$ and $\beta$ be actions of a discrete amenable group $G$. Then $\alpha$ and $\beta$ are cocycle conjugate if and only if $\Phi(\alpha)=\Phi(\beta)$ and $\bmod (\alpha)=\bmod (\beta)$. (In the type $I I_{1}$ case, the last condition is not necessary.)

Let $\varphi$ be a faithful normal state of $N$ and $E$ the minimal conditional expectation from $M$ onto $N$. Set $(\tilde{N} \subset \tilde{M}):=\left(N \rtimes_{\sigma^{\varphi}} \mathbf{R} \subset M \rtimes_{\sigma^{\varphi \circ E}} \mathbf{R}\right)$. Let $\alpha \in \operatorname{Aut}(M, N)$ and $\tilde{\alpha}$ the canonical extension of $\alpha$ to $\tilde{N} \subset \tilde{M}$. (See [7].)

Definition 2.4 ([21, Definition 3.2], [22, Definition 4.2]). Let $N \subset M$ be a subfactor type III with finite index and $\tilde{N} \subset \tilde{M} \subset \tilde{M}_{1} \subset \cdots$ be the Jones tower for $\tilde{N} \subset \tilde{M}$. For $\alpha \in$ Aut $(M, N), \alpha$ is said to be strongly free if there are no nonzero $a \in \tilde{M}_{k}$ such that $\tilde{\alpha}(x) a=a x$ holds for every $x \in \tilde{M}$. An action $\alpha$ of $G$ is said to be strongly free if $\alpha_{g}$ is strongly free for $g \in G \backslash\{e\}$.

Definition 2.5 ([22, Definition 4.2]). Set $\Upsilon(\alpha):=\left\{\left.\tilde{\alpha}\right|_{\tilde{M}^{\prime} \cap \tilde{M}_{k}}\right\}_{k}$. We call $\Upsilon(\alpha)$ the fundamental homomorphism.

In the case of subfactors of type II and type $\mathrm{III}_{\lambda}$, strong amenability has been introduced by Popa, [15] and [16]. Based on strong amenability of subfactor of type II, Winsløw introduced strong amenability for subfactors of type $\mathrm{III}_{0}$.

Definition 2.6 ([25, Definition 3.5]). Let $N \subset M$ be a subfactor of type $\mathrm{III}_{0}$ with the common flow of weights and $\tilde{N} \subset \tilde{M}:=N \rtimes_{\sigma^{\varphi}} \mathbf{R} \subset M \rtimes_{\sigma^{\varphi \circ E}} \mathbf{R}$. $N \subset M$ is said to be strongly amenable if $Z(\tilde{N})=Z(\tilde{M})$ and $(\tilde{N} \subset \tilde{M}) \cong$ $Z(\tilde{N}) \otimes(P \subset Q)$, where $P \subset Q$ is a strongly amenable subfactor of type $\mathrm{II}_{\infty}$.

On the classification of subfactors of type $\mathrm{III}_{0}$, the following result has been obtained by Winsløw.

Theorem 2.7 ([25, Theorem 4.2]). Let $N \subset M$ be a strongly amenable subfactor of type $\mathrm{III}_{0}$. Then $N \subset M$ is classified by its relative flow of weights,

$$
\mathcal{F}^{M \supset N}:=\left\{\tilde{M}_{1}^{\prime} \cap \tilde{M}_{k} \subset \tilde{M}^{\prime} \cap \tilde{M}_{k},\left.\theta_{t}\right|_{\tilde{M}^{\prime} \cap \tilde{M}_{k}}\right\}_{k} .
$$

\section{Reduction to groupoid actions.}

In the rest of this paper $N \subset M$ is always a strongly amenable subfactor of type $\mathrm{III}_{0}$ and $G$ denotes a discrete amenable group. We also use the notations in the previous section.

Our main theorem of this paper is the following. 
Theorem 3.1. Let $\alpha$ and $\beta$ be strongly free actions of $G$ on $N \subset M$. Then $\alpha$ and $\beta$ are cocycle conjugate if and only if there exists $\sigma \in \operatorname{Aut}\left(\mathcal{F}^{M \supset N}\right)$ such that

$$
\Upsilon(\alpha)=\sigma \circ \Upsilon(\beta) \circ \sigma^{-1} .
$$

We give the proof of above theorem in this section and next section.

In this section, we reduce the classification of actions of $G$ to that of actions of groupoids.

First we consider only one action $\alpha$. For the same reason as at the beginning of Section 3 in [13], we only have to classify the actions of $\tilde{G}:=G \times \mathbf{R}$ on $\tilde{N} \subset \tilde{M}$ defined by $(g, t) \rightarrow \tilde{\alpha} \theta_{t}$. We also denote this action of $\tilde{G}$ by $\tilde{\alpha}$.

Let $\left(X, \mu, \mathcal{F}_{t}\right)$ be the flow of weight of $M$. Then $L^{\infty}(X, \mu)=Z(\tilde{N})=$ $Z(\tilde{M})$. Then by assumption,

$$
\left(\tilde{N} \subset \tilde{M} \subset \tilde{M}_{1} \subset \cdots\right) \cong L^{\infty}(X, \mu) \otimes\left(P \subset Q \subset Q_{1} \subset \cdots\right)
$$

holds.

Let $\tilde{G} \ltimes X$ be the auxiliary groupoid. Then the source map $s$ and the range map $r$ from $\tilde{G} \ltimes X$ to $X$ are the following:

$$
s(g, x):=x, r(g, x):=g x, \quad(g, x) \in \tilde{G} \ltimes X .
$$

We consider the groupoid action of $\tilde{G} \ltimes X$ on $P \subset Q$ by the following equation:

$$
\tilde{\alpha}_{g}(a)=\int_{X}^{\oplus} \tilde{\alpha}_{\left(g, g^{-1} x\right)}\left(a\left(g^{-1} x\right)\right) d \mu(x) .
$$

We express $\left(X, \mu, \mathcal{F}_{t}\right)$ as the flow built under a ceiling function on the base transformation $T$ on the measure space $(Y, \nu)$. So $X$ is of the form $\{(y, s) \in Y \times \mathbf{R} \mid 0 \leq s \leq f(y)\}$. We define maps $\pi: X \rightarrow Y$ and $h: \rightarrow \mathbf{R}$ by the equality $x=(\pi(x), h(x))$. Set $\mathcal{G}:=\{\gamma \in \tilde{G} \ltimes X \mid s(\gamma), r(\gamma) \in Y\}$. The groupoid $\mathcal{G}$ is an orbitally discrete groupoid. (See [6].) For $x \in X$, put $H_{x}:=\{g \in \tilde{G} \mid g x=x\}$. Then $H_{x}$ is a discrete amenable group. Since $\mathbf{R}$ is in the center of $\tilde{G}, H_{x}=H_{t x}$ holds for $t \in \mathbf{R}$. Since $\mathbf{R}$ acts on $X$ ergodicaly, $H_{x}=H$ for a.e $x$. Then we have a semidirect product of $\mathcal{G}$ as $\mathcal{G}=H \rtimes \mathcal{K}$, where $\mathcal{K}$ is a principal groupoid derived from $\mathcal{G}$. In this case, $\mathcal{K}$ is an $\mathrm{AF}$ ergodic groupoid by [4], [26].

Here as in [13, Proposition 3.2], the following holds.

Proposition 3.2. If $\alpha$ is a strongly free action on $N \subset M$, the action of $H_{x}$ on $P \subset Q$ is strongly outer for a.e $x \in Y$.

Proof. Assume that there exists $Z \subset Y$ such that $\nu(Z)>0$ and actions of $H_{z}$ are not strongly outer for any $z \in Z$. Then for every $z \in Z$ there exists $k \in \mathbf{N}, 0 \neq a(z) \in P_{k}$ and $e \neq h \in H_{z}$ such that $\tilde{\alpha}_{h}(x) a(z)=a(z) x$ holds for every $x \in P$. It is possible that $k$ and $g$ depend on $z$. But since $H_{z}$ is 
countable, we may assume $k$ and $g$ are independent from $z \in Z$. Note that since $\tau_{Q} \tilde{\alpha}_{h}=\tau, g$ is in $G$. Set

$$
\tilde{a}:=\int_{X}^{\oplus} \tilde{\alpha}_{(h(x), \pi(x))}(a(\pi(x))) d \mu(x) .
$$

Then an easy computation shows that this element breaks strong freeness and $\alpha$ is not strongly free.

By the next lemma, we know the relation between cocycle conjugacy of actions of $\tilde{G} \ltimes X$ and that of $\mathcal{G}$.

Lemma 3.3. Let $\tilde{\alpha}$ and $\tilde{\beta}$ be actions of $\tilde{G} \ltimes X$ on $P \subset Q$. If $\tilde{\alpha}$ and $\tilde{\beta}$ are cocycle conjugate when we restrict actions on $\mathcal{G}$, then actions of $\tilde{G} \ltimes X$ are also cocycle conjugate.

Proof. The proof of [19, Theorem 4.2] works in the same way. But we present the proof here for the reader's convenience.

First we define a map from $\tilde{G} \ltimes X$ to $\mathcal{G}$ as follows

$$
p(g, x):=\left(h(g x)^{-1} g h(x), \pi(x)\right) .
$$

By assumption, there exist automorphisms $\left\{\theta_{y}\right\}_{y \in Y} \subset \operatorname{Aut}(Q, P)$ and a cocycle $v_{\gamma} \in Z_{\tilde{\beta}}^{1}(\mathcal{G}, U(P))$ such that

$$
\theta_{r(\gamma)} \circ \tilde{\alpha}_{\gamma} \circ \theta_{s(\gamma)}^{-1}=\operatorname{Ad} v_{\gamma} \tilde{\beta}_{\gamma}, \gamma \in \mathcal{G} .
$$

Define $\theta_{x}^{\prime}, x \in X$ and $v_{(g, t)}^{\prime},(g, t) \in \tilde{G} \ltimes X$ as follows:

$$
\begin{gathered}
\theta_{x}:=\tilde{\beta}_{(h(x), \pi(x))} \circ \theta_{\pi}(x) \circ \tilde{\alpha}_{(h(x), \pi(x))}^{-1}, \\
v_{(g, x)}:=\tilde{\beta}_{(h(g x), \pi(g x))}\left(v_{p(g, x)}\right) .
\end{gathered}
$$

Note that $(g, x)=(h(g x), \pi(g x)) p(g, x)(h(x), \pi(x))^{-1}$ holds. Then using the above equations, we can show that

$$
\theta_{g x}^{\prime} \circ \tilde{\alpha}_{(g, x)} \circ \theta_{x}^{\prime-1}=\operatorname{Ad} v_{(g, x)}^{\prime} \tilde{\beta}_{(g, x)}
$$

holds and this shows that actions of $\tilde{G} \ltimes X$ are cocycle conjugate.

In the end of this section, we discuss invariants of actions of $\mathcal{G}$ on $P \subset Q$.

Lemma 3.4. Let $\alpha$ and $\beta$ be actions of $G$ on $N \subset M$ such that $\Upsilon(\alpha)=$ $\Upsilon(\beta)$. Let $\tilde{\alpha}$ and $\tilde{\beta}$ be actions of $\mathcal{G}$ induced from $\alpha$ and $\beta$ respectively by the above procedure. Then we have $\Phi(\tilde{\alpha})=\Phi(\tilde{\beta})$ and $\bmod (\tilde{\alpha})=\bmod (\tilde{\beta})$.

Proof. First we compare mod. Let $\tau$ be the trace of $\tilde{M}$ such that $\tau \theta_{t}=e^{-t} \tau$, where $\theta_{t}$ is the dual action. For $(g, t) \in \tilde{G}$, set $\psi(g, t):=e^{-t}$. Then $\tau \tilde{\alpha}_{h}=$ 
$\psi(h)=\tau \tilde{\beta}_{h}$ holds for $h \in \tilde{G}$. Express $\tau$ by direct integral, $\tau=\int_{X}^{\oplus} \tau_{x} d \mu(x)$. Then

$$
\begin{aligned}
\tau \tilde{\alpha}_{h}(a) & =\int_{X}^{\oplus} \tau_{h x}\left(\tilde{\alpha}_{(h, x)}(a(x))\right) d \mu(g x) \\
& =\int_{X}^{\oplus} \tau_{h x}\left(\tilde{\alpha}_{(h, x)}(a(x))\right) \frac{d \mu \circ g}{d \mu}(x) d \mu(x)
\end{aligned}
$$

holds and we get an equality $d \mu \circ g / d \mu(x) \tau_{h x} \tilde{\alpha}_{(h, x)}=e^{-t} \tau_{x}$. A similar equality holds for $\tilde{\beta}$. From these equations, we can easily get $\bmod \left(\tilde{\alpha}_{(h, x)}\right)=$ $\bmod \left(\tilde{\beta}_{(h, x)}\right)$.

Next we compare $\Phi$. By assumption, $\left.\tilde{\alpha}_{h}\right|_{\tilde{M}^{\prime} \cap \tilde{M}_{k}}=\left.\tilde{\beta}_{h}\right|_{\tilde{M}^{\prime} \cap \tilde{M}_{k}}$ holds for every $k$. Note that an action $\tilde{\alpha}$ of $\tilde{G} \ltimes X$ can be extended to $Q_{k}$ such that the equation

$$
\tilde{\alpha}_{h}(a)=\int_{X}^{\oplus} \tilde{\alpha}_{\left(h, h^{-1} x\right)}\left(a\left(h^{-1} x\right)\right) d \mu(x)
$$

holds for every $a \in \tilde{M}_{k}$. (See the proof of [25, Lemma 4.3].) Then we get an equality $\Phi(\tilde{\alpha})=\Phi(\tilde{\beta})$.

\section{Classification results.}

Let $\alpha$ and $\beta$ be strongly free actions of $G$ on $N \subset M$. By the results of previous section, we get two actions $\tilde{\alpha}$ and $\tilde{\beta}$ of a groupoid $\mathcal{G}$ such that $\tilde{\alpha}_{h}$ and $\tilde{\beta}_{h}$ act strongly freely on $P \subset Q$ and satisfy $\Phi(\tilde{\alpha})=\Phi(\tilde{\beta})$ and $\bmod (\tilde{\alpha})=\bmod (\tilde{\beta})$.

In this section, we classify actions of $\mathcal{G}$ on $P \subset Q$ and complete the classification of actions of $G$ on $N \subset M$.

Theorem 4.1. Two actions $\tilde{\alpha}$ and $\tilde{\beta}$ of $\mathcal{G}$ are cocycle conjugate, i.e., there exists $\left\{\theta_{y}\right\}_{y \in Y} \subset \operatorname{Aut}(Q, P)$ and a cocycle $u_{\gamma} \in Z_{\tilde{\beta}}^{1}(\mathcal{G}, U(P))$ such that

$$
\theta_{r(\gamma)} \circ \tilde{\alpha}_{\gamma} \circ \theta_{s(\gamma)}^{-1}=\operatorname{Ad} u_{\gamma} \tilde{\beta}_{\gamma}, \quad \gamma \in \mathcal{G} .
$$

In the following, we give a proof of Theorem 4.1.

We express $\mathcal{G}$ as the semidirect product $H \rtimes \mathcal{K}$. We use Theorem 2.3 to classify the $H$ part and the cohomology lemma to classify the $\mathcal{K}$ part.

First we compare the $H$ part. By the above assumption, we have $\Phi\left(\tilde{\alpha}_{h}\right)=$ $\Phi\left(\tilde{\beta}_{h}\right)$ and $\bmod \left(\tilde{\alpha}_{h}\right)=\bmod \left(\tilde{\beta}_{h}\right)$ for $h \in H_{x}$. So by Theorem 2.3 , there exist automorphisms $\left\{\theta_{x}\right\}_{x \in Y}$ and a cocycle $u_{h} \in Z_{\tilde{\beta}}^{1}\left(H_{x}, U(P)\right)$ such that

$$
\theta_{x} \circ \tilde{\alpha} \circ \theta_{x}^{-1}=\operatorname{Ad} u_{h} \tilde{\beta}_{h} .
$$

So we replace $\tilde{\alpha}_{\gamma}$ by $\theta_{r(\gamma)} \circ \tilde{\alpha} \circ \theta_{s(\gamma)}^{-1}$, we may assume $\tilde{\alpha}_{h}=\operatorname{Ad} u_{h} \tilde{\beta}_{h}$ for $h \in H_{x}$. 
Next we compare the $\mathcal{K}$ part by applying the cohomology lemma, $[\mathbf{1 7}$, Theorem 5.5]. To use the cohomology lemma, we need several preparations.

Define two groups $N_{x}^{0}$ and $N_{x}^{1}$ as follows:

$$
\begin{aligned}
N_{x}^{1}: & =\left\{\left(\theta, v_{h}\right) \mid \theta \in \operatorname{Aut}(Q, P), \tau_{Q} \theta=\tau_{Q}, \Phi(\theta)=\mathrm{id},\right. \\
& \left.v_{h} \in Z_{\tilde{\beta}}^{1}\left(H_{x}, U(P)\right), \theta \circ \tilde{\beta}_{h} \circ \theta^{-1}=\operatorname{Ad} v_{h} \tilde{\beta}_{h}, \text { for } h \in H_{x}\right\}, \\
N_{x}^{0}: & =\left\{\left(\operatorname{Ad} u, u \tilde{\beta}_{h}\left(u^{*}\right)\right) \mid u \in U(P), h \in H_{x}\right\} .
\end{aligned}
$$

Then obviously $N_{x}^{0} \subset N_{x}^{1}$.

Lemma 4.2. $N_{x}^{0}$ is dense in $N_{x}^{1}$.

Proof. Take $\left(\theta, v_{h}\right) \in N_{x}^{1}$. Since $\Phi(\theta)=\mathrm{id}$ and $\tau_{Q} \theta=\tau, \theta$ is $\operatorname{in} \overline{\operatorname{Int}}(Q, P)$. (See [22].) So there exist $\left\{u_{n}\right\} \in U(P)$ such that $\lim _{n \rightarrow \infty} \operatorname{Ad} u_{n}=\theta$. By assumption, $\theta \circ \tilde{\beta}_{h} \circ \theta^{-1}=\operatorname{Ad} v_{h} \tilde{\beta}_{h}$ holds, so $\lim _{n \rightarrow \infty} \operatorname{Ad} u_{n} \tilde{\beta}_{h}\left(u_{n}^{*}\right) \tilde{\beta}_{h}=$ Ad $v_{h} \tilde{\beta}_{h}$ holds and $\left\{v_{h}^{*} u_{n} \tilde{\beta}_{h}\left(u_{n}^{*}\right)\right\}_{n}$ is a central sequence.

Fix a free ultrafilter $\omega$ over $\mathbf{N}$. Let $\mathcal{C}_{\omega}(Q, P)$ be the central sequence algebra for $P \subset Q$ and $\tilde{\beta}^{(\omega)}$ the induced action on $\mathcal{C}_{\omega}(Q, P)$ from $\tilde{\beta}$. Then strong outerness of the action $\tilde{\beta}$ on $P \subset Q$ means that $\tilde{\beta}^{(\omega)}$ acts freely on $\mathcal{C}_{\omega}(Q, P)$. (See [21, Proposition 3.4] and [2, Proposition 2.1.2].)

Here we need the next proposition, which is a subfactor version of the 1-cohomology vanishing theorem of [14, Proposition 7.2].

Proposition 4.3. Every 1-cocycle $w_{h} \in Z_{\tilde{\beta}(\omega)}^{1}\left(H_{x}, U\left(\mathcal{C}_{\omega}(Q, P)\right)\right)$ is a coboundary, i.e., there exists a unitary $w^{\prime}$ in $\mathcal{C}_{\omega}(Q, P)$ such that $w^{\prime *} \tilde{\beta}_{h}^{(\omega)}\left(w^{\prime}\right)=$ $w_{h}$.

The proof in [14] works in the same way. When the group is $\mathbf{Z}$, the above result has been obtained by Loi in [12, Proposition 4.2]. We can easily verify that $\left\{v_{h}^{*} u_{n} \tilde{\beta}_{h}\left(u_{n}^{*}\right)\right\}_{n}$ is a $\tilde{\beta}^{\omega}$ cocycle. So by Proposition 4.3 , we can find a unitary $w=\left\{w_{n}\right\}_{n} \in \mathcal{C}_{\omega}(Q, P)$ such that $\left\{w_{n}^{*} \tilde{\beta}_{h}\left(w_{n}\right)\right\}_{n}=\left\{v_{h}^{*} u_{n} \tilde{\beta}_{h}\left(u_{n}^{*}\right)\right\}_{n}$ in $\mathcal{C}_{\omega}(Q, P)$. Set $y_{n}:=w_{n} u_{n}$. Then $\lim _{n \rightarrow \omega} \operatorname{Ad} y_{n}=\theta$ holds, and

$$
\begin{aligned}
\lim _{n \rightarrow \omega} y_{n} \tilde{\beta}_{h}\left(y_{n}^{*}\right) & =\lim _{n \rightarrow \omega} w_{n} u_{n} \tilde{\beta}_{h}\left(u_{n}^{*} w_{n}^{*}\right) \\
& =\lim _{n \rightarrow \omega} v_{h} v_{h}^{*} w_{n} u_{n} \tilde{\beta}_{h}\left(u_{n}^{*} w_{n}^{*}\right) \\
& =\lim _{n \rightarrow \omega} v_{h} w_{n} v_{h}^{*} u_{n} \tilde{\beta}_{h}\left(u_{n}^{*}\right) \tilde{\beta}_{h}\left(w_{n}^{*}\right) \\
& =\lim _{m \rightarrow \omega} v_{h} w_{n} w_{n}^{*} \tilde{\beta}_{h}\left(w_{n}\right) \tilde{\beta}_{h}\left(w_{n}^{*}\right) \\
& =v_{h} .
\end{aligned}
$$

So by choosing a subsequence, we get unitaries $\left\{y_{n}\right\}_{n}$ such that

$$
\lim _{n \rightarrow \infty}\left(\operatorname{Ad} y_{n}, y_{n} \tilde{\beta}_{h}\left(y_{n}^{*}\right)\right)=\left(\theta, v_{h}\right) .
$$


This shows that $N_{x}^{0}$ is dense in $N_{x}^{1}$ and the proof of Lemma 4.2 is complete.

For $k=(y, x) \in \mathcal{K}$, put $\gamma_{k}:=\tilde{\alpha}_{k} \tilde{\beta}_{k}^{-1}$. Then it is clear that $\Phi\left(\gamma_{k}\right)=\mathrm{id}$ and $\bmod \left(\gamma_{k}\right)=1$. Moreover

$$
\begin{aligned}
\gamma_{k} \tilde{\beta}_{h} \gamma_{k}^{-1} & =\tilde{\alpha}_{k} \tilde{\beta}_{k^{-1} h k} \tilde{\alpha}_{k}^{-1} \\
& =\tilde{\alpha}_{k} \operatorname{Ad} u_{k^{-1} h k}^{*} \tilde{\alpha}_{k^{-1} h k} \tilde{\alpha}_{k}^{-1} \\
& =\operatorname{Ad} \tilde{\alpha}_{k}\left(u_{k^{-1} h k}^{*}\right) \tilde{\alpha}_{h} \\
& =\operatorname{Ad} \tilde{\alpha}_{k}\left(u_{k^{-1} h k}^{*}\right) u_{h} \tilde{\beta}_{h}
\end{aligned}
$$

holds and $\tilde{\alpha}_{k}\left(u_{k^{-1} h k}^{*}\right) u_{h}$ is a $\tilde{\beta}$ cocycle. So $\tilde{\gamma}_{k}:=\left(\gamma_{k}, \tilde{\alpha}_{k}\left(u_{k^{-1} h k}^{*} u_{h}\right)\right)$ is an element of $N_{x}^{1}$.

Next, for $k=(y, x) \in \mathcal{K}$, we define a Borel map $N_{k}^{1}$ from $N_{x}^{1}$ to $N_{y}^{1}$ as follows:

$$
N_{k}^{1}\left(\theta, v_{h}\right):=\left(\tilde{\beta}_{k} \circ \theta \circ \tilde{\beta}_{k}^{-1}, \tilde{\beta}\left(v_{k^{-1} h k}\right)\right) .
$$

Then $\left(N_{x}^{1}, N_{k}^{1}\right)$ is a Borel functor in the sense of [17, Definition 4.1] and $\tilde{\gamma}$ is a $N_{k}^{1}$ cocycle, i.e., $\tilde{\gamma}_{k_{1} k_{2}}=\tilde{\gamma}_{k_{1}} N_{k_{1}}^{1}\left(\tilde{\gamma}_{k_{2}}\right)$. Here we apply the cohomology lemma, [17, Theorem 5.5], for $\tilde{\gamma}$ and id .

Then we get Borel maps $\theta: Y \rightarrow$ Aut $(Q, P)$ and $a: \mathcal{K} \rightarrow U(P)$ and a cocycle $v_{h} \in Z_{\tilde{\beta}}^{1}\left(H_{x}, U(P)\right)$ which satisfy equalities

$$
\begin{gathered}
\theta_{y} \circ \gamma_{k} \circ \theta_{x}^{-1}=\operatorname{Ad} a_{k}, \quad k=(y, x) \in \mathcal{K}, \\
\theta_{x} \circ \tilde{\beta}_{h} \circ \theta_{x}^{-1}=\operatorname{Ad} v_{h} \tilde{\beta}_{h} .
\end{gathered}
$$

Since $\mathcal{K}$ is $\mathrm{AF}$, we may consider that $a_{k}$ is a cocycle for $\tilde{\beta}_{k}$. By definition of $\gamma_{k}$, we obtain the equality

$$
\theta_{y} \circ \tilde{\alpha}_{k} \circ \theta_{x}^{-1}=\operatorname{Ad} a_{k} \tilde{\beta}_{k} .
$$

Then for $h \in H_{y}$ and $k=(y, x) \in \mathcal{K}$

$$
\begin{aligned}
\theta_{y} \circ \tilde{\alpha}_{h k} \circ \theta_{x}^{-1} & =\theta_{y} \circ \tilde{\alpha}_{h} \circ \theta_{y}^{-1} \circ \theta_{y} \circ \tilde{\alpha}_{k} \circ \theta_{x}^{-1} \\
& =\theta_{y} \circ \operatorname{Ad} u_{h} \circ \tilde{\beta}_{h} \circ \theta_{y}^{-1} \circ \operatorname{Ad} a_{k} \circ \tilde{\beta}_{k} \\
& =\operatorname{Ad} \theta_{y}\left(u_{h}\right) v_{h} \circ \tilde{\beta}_{h} \circ \operatorname{Ad} a_{k} \circ \tilde{\beta}_{k} \\
& =\operatorname{Ad} \theta_{y}\left(u_{h}\right) v_{h} \tilde{\beta}_{h}\left(a_{k}\right) \tilde{\beta}_{h k} .
\end{aligned}
$$

Set $a_{h}:=\theta_{x}\left(u_{h}\right) v_{h}$ for $h \in H_{x}$ and $a_{h k}:=a_{h} \tilde{\beta}_{h}\left(a_{k}\right)$, we get

$$
\theta_{y} \circ \tilde{\alpha}_{h k} \circ \theta_{x}=\operatorname{Ad} a_{h k} \tilde{\beta}_{h k} .
$$

Here $a_{h}$ and $a_{k}$ are cocycles for $\tilde{\beta}_{h}$ and $\tilde{\beta}_{k}$, but $a_{h k}$ is not necessary a cocycle for $\tilde{\beta}_{h k}$. But by comparing $\tilde{\alpha}_{h_{1} k_{1}} \tilde{\alpha}_{h_{2} k_{2}}$ with $\tilde{\alpha}_{h_{1} k_{1} h_{2} k_{2}}$, we get the following equality:

$$
a_{h_{1} k_{1}} \tilde{\beta}_{h_{1} k_{1}}\left(a_{h_{2} k_{2}}\right)=\varphi\left(k_{1}, h_{2}\right) a_{h_{1} k_{1} h_{2} k_{2}},
$$


where $\varphi\left(k_{1}, h_{2}\right)$ is the element of $Z^{1}(\mathcal{K}, \hat{H})$ and does not depend on $h_{1}$ and $k_{2}$, where $\hat{H}$ is the set of 1 dimensional representations of $H$.

To remove $\varphi$, we use the model actions constructed by Sutherland and Takesaki.

Theorem 4.4 ([18, Theorem 3.1]). Let $R_{0,1}$ be the AFD factor of type $\mathrm{II}_{\infty}$. Then there exists an action $m$ of $\mathcal{G}$ satisfy the following conditions.

(1) For every $y \in Y, H_{y}$ acts outer on $R_{0,1}$.

(2) $\bmod (m)=\bmod (\tilde{\alpha})$.

(3) For every $\varphi \in Z^{1}(\mathcal{K}, \hat{H})$, there exist $\left\{\theta_{y}\right\}_{y \in Y} \subset \operatorname{Aut}\left(R_{0,1}\right)$ and $u_{h k} \in$ $U\left(R_{0,1}\right)$ such that:

(a) $u_{h}$ and $u_{k}$ are cocycle for $H_{y}$ and $\mathcal{K}$,

(b) $\theta_{y} \circ m_{h k} \circ \theta_{x}=\operatorname{Ad} u_{h k} m_{h k}$,

(c) $u_{h_{1} k_{1}} m_{h_{1} k_{1}}\left(u_{h_{2} k_{2}}\right)=\overline{\varphi\left(k_{1}, h_{2}\right)} u_{h_{1} k_{1} h_{2} k_{1}}$.

We define the model action $\bar{m}$ of $\mathcal{G}$ on $(P \subset Q) \cong\left(\vee_{k}\left(P_{k}^{\prime} \cap P\right) \subset \vee_{k}\left(P_{k}^{\prime} \cap\right.\right.$ $Q)) \otimes R_{0,1}$ as follows:

$$
\bar{m}_{\gamma}:=\tilde{\alpha}_{\gamma}^{s t} \otimes m_{\gamma}
$$

We complete the proof of Theorem 4.1 by comparing $\tilde{\alpha}$ with the model action $\bar{m}$.

The action $\bar{m}$ has the same invariant of $\tilde{\alpha}$ and $\bar{m}_{h}, h \in H_{x}$, acts strongly outer on $P \subset Q$ by the construction of $\bar{m}$. Then the above discussion is valid for $\tilde{\alpha}$ and $\bar{m}$, so we may assume the following equalities:

$$
\begin{gathered}
\tilde{\alpha}_{h k}=\operatorname{Ad} a_{h k} \circ \bar{m}_{h k}, \\
a_{h_{1} k_{1}} \bar{m}_{h_{1} k_{1}}\left(a_{h_{2} k_{2}}\right)=\varphi\left(k_{1}, h_{2}\right) a_{h_{1} k_{1} h_{2} k_{2}}
\end{gathered}
$$

for some $\varphi$. Take $\left\{\theta_{y}\right\}_{y \in Y} \subset \operatorname{Aut}\left(R_{0,1}\right)$ and $u_{h k}$ satisfying the conditions of Theorem 4.4.

Then

$$
\begin{aligned}
& \left(\mathrm{id} \otimes \theta_{r(k)}\right) \circ \tilde{\alpha}_{h k} \circ\left(\mathrm{id} \otimes \theta_{s(k)}\right)^{-1} \\
& =\left(\mathrm{id} \otimes \theta_{r(k)}\right) \circ \operatorname{Ad} a_{h k} \circ \bar{m}_{h k} \circ\left(\mathrm{id} \otimes \theta_{s(k)}\right)^{-1} \\
& =\operatorname{Ad}\left(\mathrm{id} \otimes \theta_{r(k)}\right)\left(a_{h k}\right) \tilde{\alpha}_{h k}^{s t} \otimes\left(\theta_{r(k)} \circ m_{h k} \circ \theta_{s(k)}^{-1}\right) \\
& =\operatorname{Ad}\left(\mathrm{id} \otimes \theta_{r(k)}\right)\left(a_{h k}\right) \tilde{\alpha}_{h k}^{s t} \otimes\left(\operatorname{Ad} u_{h k} m_{h k}\right) \\
& =\operatorname{Ad}\left(\mathrm{id} \otimes \theta_{r(k)}\right)\left(a_{h k}\right)\left(1 \otimes u_{h k}\right) \bar{m}_{h k}
\end{aligned}
$$


and

$$
\begin{aligned}
& \left(\mathrm{id} \otimes \theta_{r\left(k_{1}\right)}\right)\left(a_{h_{1} k_{1}}\right)\left(1 \otimes u_{h_{1} k_{1}}\right) \bar{m}_{h_{1} k_{1}}\left(\left(\mathrm{id} \otimes \theta_{r\left(k_{2}\right)}\right)\left(a_{h_{2} k_{2}}\right)\left(1 \otimes u_{h k}\right)\right) \\
& =\left(\mathrm{id} \otimes \theta_{r\left(k_{1}\right)}\right)\left(a_{h_{1} k_{1}}\right)\left(\tilde{\alpha}_{h_{1} k_{1}}^{s t} \otimes \theta_{r\left(k_{1}\right)} m_{h_{1} k_{1}}\right)\left(a_{h_{2} k_{2}}\right)\left(1 \otimes u_{h_{1} k_{1}}\right) \\
& \quad \cdot\left(1 \otimes m_{h_{1} k_{1}}\left(u_{h_{2} k_{2}}\right)\right) \\
& =\left(\mathrm{id} \otimes \theta_{r\left(k_{1}\right)}\right)\left(a_{h_{1} k_{1}} \bar{m}_{h_{1} k_{1}}^{s t}\left(a_{h_{2} k_{2}}\right)\right)\left(1 \otimes u_{h_{1} k_{1} m_{h_{1} k_{1}}}\left(u_{h_{2} k_{2}}\right)\right) \\
& =\left(\mathrm{id} \otimes \theta_{r\left(k_{1}\right)}\right)\left(\varphi\left(k_{1}, h_{2}\right) a_{h_{1} k_{1} h_{2} k_{2}}\right)\left(1 \otimes \overline{\varphi\left(k_{1}, h_{2}\right)} u_{h_{1} k_{1} h_{2} k_{2}}\right) \\
& =\left(\mathrm{id} \otimes \theta_{r\left(k_{1}\right)}\right)\left(a_{h_{1} k_{1} h_{2} k_{2}}\right) u_{h_{1} k_{1} h_{2} k_{2}}
\end{aligned}
$$

hold, so (id $\left.\otimes \theta_{r(k)}\right)\left(a_{h k}\right)\left(1 \otimes u_{h k}\right)$ is a cocycle for $\bar{m}_{h k}$ and finally we can conclude two actions $\tilde{\alpha}$ and $\bar{m}$ are cocycle conjugate and proof of Theorem 4.1 is complete.

Proof of Theorem 3.1. Let $\alpha$ and $\beta$ be strongly free actions of $G$ on $N \subset M$ such that $\Upsilon(\alpha)=\Upsilon(\beta)$. Then by the results of Section 3 and Section 4 , two actions $\tilde{\alpha}$ and $\tilde{\beta}$ of $\mathcal{G}$ on $P \subset Q$ are cocycle conjugate. Then by Lemma 3.3, two actions $\tilde{\alpha}$ and $\tilde{\beta}$ are cocycle conjugate, i.e., there exist automorphisms $\left\{\theta_{x}\right\}_{x \in X} \subset \operatorname{Aut}(Q, P)$ and a cocycle $u_{\gamma} \in Z_{\tilde{\beta}}(\tilde{G} \ltimes X, U(P))$ such that

$$
\theta_{r(\gamma)} \circ \tilde{\alpha}_{\gamma} \circ \theta_{s(\gamma)}^{-1}=\operatorname{Ad} u_{\gamma} \tilde{\beta}_{\gamma} .
$$

We put $\tilde{\theta}:=\int_{X}^{\oplus} \theta_{x} d \mu(x)$ and $u_{g}:=\int_{X}^{\oplus} u_{(g, x)} d \mu(x)$, then we get

$$
\tilde{\theta} \circ \tilde{\alpha}_{g} \circ \tilde{\theta}^{-1}=\operatorname{Ad} u_{g} \tilde{\beta}_{g}
$$

for $g \in \tilde{G}$, so actions $\tilde{\alpha}$ and $\tilde{\beta}$ of $\tilde{G}=G \times \mathbf{R}$ are cocycle conjugate and we conclude $\alpha$ and $\beta$ are cocycle conjugate as explained at the beginning of Section 3.

\section{References}

[1] M. Choda and H. Kosaki, Strongly outer actions for an inclusion of factors, J. Funct. Anal., 122 (1994), 315-332.

[2] A. Connes, Outer conjugacy classes of automorphisms of factors, Ann. Sci. Ec. Nor. Sup., 8 (1975), 383-419.

[3] _ Classification of injective factors, Ann. Math., 104 (1976), 73-115.

[4] A. Connes, J. Feldman and B. Weiss, An amenable equivalence relation is generated by a single transformation, Ergod. Th. and Dynam. Sys., 1 (1981), 431-450.

[5] A. Connes and M. Takesaki, The flow of weights on factors of type III, Tohoku. Math. J., 29 (1977), 473-555.

[6] J. Feldman, P. Hahn and C. Moore, Orbit structure and countable sections for actions of continuous groups, Adv. Math., 28 (1978), 186-230.

[7] U. Haagerup and E. Størmer, Equivalences of normal states on von Neumann algebras and the flow of weights, Adv. Math., 83 (1990), 180-262. 
[8] V.F.R. Jones, Index for subfactors, Invent. Math., 72 (1983), 1-25.

[9] V.F.R. Jones and M. Takesaki, Actions of compact abelian groups on semifinite injective factors, Acta. Math., 153 (1984), 213-258.

[10] Y. Kawahigashi, C. Sutherland and M. Takesaki, The structure of the automorphism group of an injective factor and the cocycle conjugacy of discrete abelian group actions, Acta. Math., 169 (1992), 105-130.

[11] W. Krieger, On ergodic flows and isomorphism of factors, Math. Ann., 223 (1976), 19-70.

[12] P. Loi, On Automorphisms of subfactors, J. Funct. Anal., 141 (1996), 275-293.

[13] T. Masuda, Classification of actions of discrete amenable groups on strongly amenable subfactors of type $\mathrm{III}_{\lambda}$, Proc. Math. Amer. Math. Soc., 127 (1999), 2053-2057.

[14] A. Ocneanu, Actions of discrete amenable groups on von Neumann algebras, Lecture Notes in Math., 1138, Springer, Berlin, (1985).

[15] S. Popa, Classification of amenable subfactors of type II, Acta. Math., 172 (1994), 163-225.

[16] Classification of actions of discrete amenable groups on amenable subfactors of type II, preprint, 1992.

[17] C. Sutherland, A Borel parametrization of Polish groups, Publ. RIMS., 21 (1985), 1067-1086.

[18] C. Sutherland and M. Takesaki, Actions of discrete amenable groups and groupoids on von Neumann algebras, Publ. RIMS., 21 (1985), 1087-1120.

[19] Actions of discrete amenable groups on injective factors of type $\mathrm{III}_{\lambda}, \lambda \neq 1$, Pac. J. Math., 137 (1989), 405-444.

[20] M. Takesaki, Duality for crossed products and the structure of von Neumann algebras of type III, Acta. Math., 131 (1973), 249-310.

[21] C. Winsløw, Strongly free actions on subfactors, Internat. J. Math., 4 (1993), 675-688.

$[22] \_$, Approximately inner automorphisms on inclusions of type $\mathrm{III}_{\lambda}$ factors, Pac. J. Math., 166 (1994), 385-400.

[23] - Automorphism on an inclusion of factors, Subfactors, Proceeding of the Taniguchi Symposium, Katata, (eds. H. Araki, et al.), World Scientific, (1994), 139152.

[24] The flow of weights in subfactor theory, Publ. RIMS., 30 (1995), 519-532.

[25] _ Classification of strongly amenable subfactors of type $\mathrm{III}_{0}$, J. Funct. Anal., 148 (1997), 296-313.

[26] R. Zimmer, Amenable ergodic group actions and an application to Poisson boundaries of random walks, J. Funct. Anal., 27 (1978), 350-372.

Received June 1, 1997.

Kouch University

KOUCH, 780-8520

JAPAN

E-mail address: masuda@math.kochi-u.ac.jp 